\title{
3D-Microstructuring of Sapphire using fs-Laser Irradiation and Selective Etching
}

\author{
Maren Hörstmann-Jungemann, Jens Gottmann and Martin Keggenhoff \\ Lehrstuhl für Lasertechnik (LLT), RWTH Aachen University, Steinbachstraße 15, 52074 Aachen, \\ Germany \\ E-mail:maren.hoerstmann-jungemann@llt.rwth-aachen.de
}

\begin{abstract}
We demonstrate a technique called "In volume Selective Laser Etching” (ISLE) for fs laser micro structuring of sapphire with subsequent wet etching in HF acid for the formation of microchannels and hollow volumes. In the process the sapphire sample is irradiated and by that modified with tightly focused fs-laser radiation as well on the surface as in the volume. Afterwards the sample is etched for about 48 hours in a $48 \%$ hydrofluoric acid to remove the modified material. Due to the non-linear absorption process in transparent materials a complete variability in three dimensions is given. This allows the modification also in the volume of sapphire samples for the fabrication of, e.g micro channels and hollow volumes,, which are needed for micro fluidic applications. More over ISLE can be used as a kind of cutting process to fabricate 3D micromechanical devices with high precision and without after treatment like polishing. The average surface roughness which can be reached with this method is $64 \mathrm{~nm}$.
\end{abstract}

DOI:10.2961/jlmn.2010.02.0009

Keywords: Fs-direct writing, selective wet etching, sapphire, fs-laser, micro structuring

\section{Content}

Micro structuring of sapphire and fused silica [1] is an important market. The reduction of time and material loss is still a challenge for the production of micromechanical parts in sapphire. New techniques for the processing of sapphire to enable for example the production of microfluidic devices [4] and sensors that combine optical and microfluidic functions [2] are requested.

The direct machining of dielectrics using short pulsed laser radiation is a promising technique for the fabrication of 3D photonic devices. A combined approach of material modification followed by chemical wet etching provides the possibility of manufacturing 3D microchannels or surface structures for integrated optical or microfluidic devices $[5,6,7,8]$. The capability for the in-volume material processing in 3 dimensions originates from the non-linear absorption of light in transparent material. The required intensities in excess of $10^{14} \mathrm{~W} / \mathrm{cm}^{2}$ for most of the dielectrics resulting in a highly non-linear light-matter interaction can be achieved by tightly focusing of the ultra short laser pulse [10]. The use of laser pulses shorter than the electronphonon coupling time (several psec) minimizes the heating. And repetition rates $\mathrm{f}<500 \mathrm{kHz}$ avoids heat accumulation effects. The modified area can be localized and controlled on a micrometer scale, while the surrounded material remains unaffected by the laser beam.

Etching ratios between the modified and the unmodified material of 100:1 in glass and more than 10,000:1 in sapphire have been shown $[1,9,10]$.

Here we present a laser assisted process called Involume Selective Laser Etching (ISLE). The process consists of two steps.
First, the sapphire is irradiated and by that modified with tightly focused fs-laser radiation. With this process we take advantage of the non-linear absorption of sapphire for the laser wavelength of $\lambda=1045 \mathrm{~nm}$. Moreover we also use the fact that due to local modification by tightly focusing $(\mathrm{N} . \mathrm{A}=0.6)$ we can not only process the material on the surface but also in the volume without damaging the surface.

Second, the sample is etched for about 48 hours in a $48 \%$ hydrofluoric acid [11] to remove the modified material and reveal hollow micro channels, hollow volumes or remove complete micromechanical devices, which were surrounded by modified material. $\mathrm{KOH}$ also can be used as a solvent for the wet etching step [3], but will not be presented here.

Here ISLE is used for the crack free combination of several microchannels on top or next to each other. The parameters used in this work can be applied for cutting micro devices out of sapphire or producing hollow volumes. It was found that the achievable average surface roughness is with $64 \mathrm{~nm}$ already suitable for mechanical devices. Beside this the material losses are minimized with ISLE.

\section{Experimental}

The irradiation is carried out with an Yb-glass fiber chirped pulse amplifier (FCPA) IMRA $\mu$ Jewel with a central wavelength of $\lambda=1045 \mathrm{~nm}$, a pulse duration $\tau=450 \mathrm{fs}$ and a variable repetition rate $\mathrm{f}=100 \mathrm{kHz}-3 \mathrm{MHz}$ (in this work a repetition rate of $\mathrm{f}=500 \mathrm{kHZ}$ was used). The maximum average power of $\mathrm{P}_{\mathrm{av}}=1.5 \mathrm{~W}$ is adjustable using a half wavelength retardation plate and a linear polarizer (Fig. 
1). The laser beam is transferred into a microscope objective with a numerical aperture of $\mathrm{NA}=0.6$. The microscope objective allows an adjustable compensation of the spherical aberration for $0-2 \mathrm{~mm}$ cover glass thickness. The spherical aberration compensation allows to keep a constant length of the focus of $2 \mathrm{z}_{\mathrm{R}}=1.85 \mu \mathrm{m}$ in $\mathrm{z}$-direction (Fig. 1) and by that a high precisions. The linear spot size (ydirection) of the focus is approximately $1 \mu \mathrm{m}$.

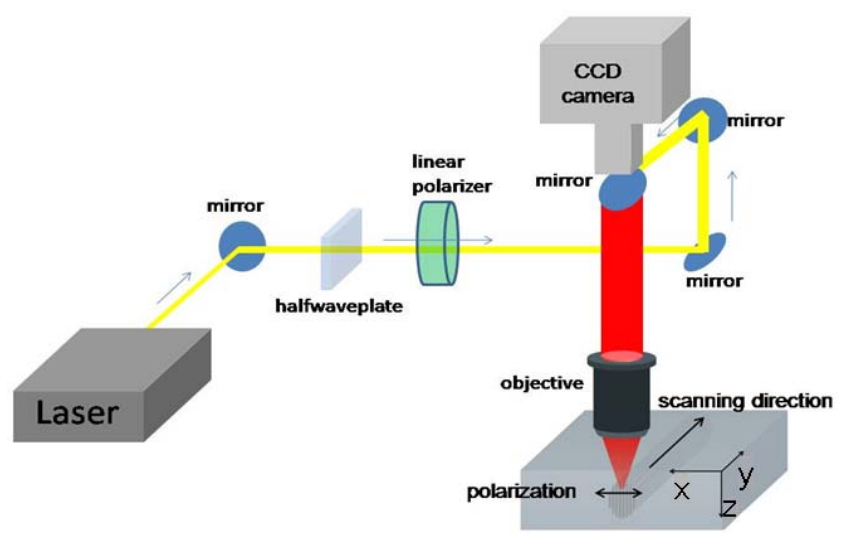

Fig. 1: Experimental setup

The sample is mounted on a programmable three axis translation stage (accuracy $\Delta \mathrm{x}=100 \mathrm{~nm}$, maximum speed $\mathrm{v}_{\max }=2 \mathrm{~mm} / \mathrm{s}$ ). The used writing speed is $\mathrm{v}=1 \mathrm{~mm} / \mathrm{s}$. Since the writing speed is significantly small compared to the repetition rate of the laser, the pulse to pulse overlap is large and the laser-affected zones can be considered as lines along the scanning direction.

The used material is a $10 \mathrm{~mm} \times 10 \mathrm{~mm} \times 1 \mathrm{~mm}$ sapphire sample with the crystalline orientation of (0001). Top and bottom surface are polished to optical quality.

The irradiation is carried out in ambient air.

\subsection{Sample processing (ISLE)}

The samples have been cleaned in an ultrasonic bath of ethanol before irradiation to make sure that the laser beam will not be scattered at small particles.

Processing steps:
$\begin{aligned} & \text { 1) fs-laser in-volume modification } \\ & \Rightarrow \text { Etch preparation: lapping }\end{aligned}$

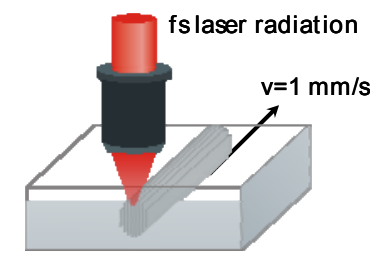

2) etching for $24 \mathrm{~h}$ in $48 \%$ aqueous solution of HF acid

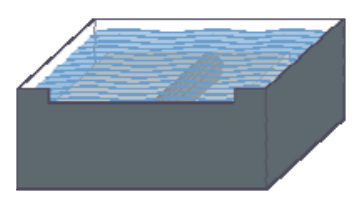

Fig. 2: Sketch of the two step process In-Volume Selective Laser Etching (ISLE).

After irradiation (step 1) (Fig. 2 top) the sample is prepared for etching (step2) (Fig. 2 bottom). For etching the acid need to be in contact with the modified sapphire. If that is not already given by the irradiation strategy the contact will be established by lapping the unmodified sapphire away.

\subsection{Principle of selective laser induced etching}

In-Volume Selective Laser Etching (ISLE) is a two step process.
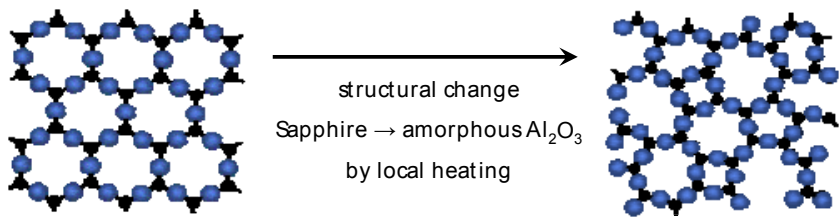

Fig. 3: The crystalline structure of sapphire is changed to an amorphous $\mathrm{Al}_{2} \mathrm{O}_{3}$ due to local heating because of nonlinear absorption of the laser beam.

First the sample is modified by tightly focused fs-laser radiation (Fig. 2 top). Due to the non linear absorption e.g. multiphoton absorption and avalanche absorption in transparent dielectrics energy is deposited in the material if the intensities are high enough (about $\mathrm{I}=10^{13} \mathrm{~W} / \mathrm{cm}^{2}$ ). Using sapphire the deposition of energy in the crystalline structure leads to an amorphisation [9] (Fig. 3). The amorphous sapphire has compared to sapphire a lower refractive index (Fig. 4).

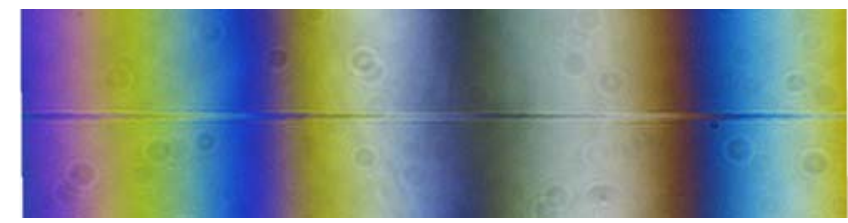

Fig. 4: White light microscopy image shows the refractive index difference between sapphire and amorphous $\mathrm{Al}_{2} \mathrm{O}_{3}$. The line in the center is the irradiated area.

The etching ratio between sapphire and amorphous sapphire is $1: 10,000$ [13].

This property is used during the second process step to remove the amorphous sapphire by immersing the sample for 24-48 hours in 48\% HF acid (Fig. 2 bottom). The etching process is carried out in an ultrasonic bath to provide a permanent movement of high concentrated acid to the modified sapphire.

The above described process is improved by using microscope objectives with spherical aberration compensation. Due to the change in focal spot size caused by spherical aberration the laser intensity varies with the working depth. Adjusting energies far above the ablation threshold on the surface mentioned earlier leads to cracks in the material if the working depth is changed. A better solution is to control the spherical aberration for every working height and work at the ablation threshold. For samples (sapphire) with a thickness of $500 \mu \mathrm{m}$ and less it turned out that a compensation of the spherical aberration for the starting depth (samples are always modified from bottom to top) and work with pulse energies of $E_{p}=24 \mu \mathrm{J}$ is suitable. If the absorption process is once started lower energies due to spherical aberration are not a problem. 


\subsection{Sample inspection}

Before etching the size of the laser affected zone is investigated. Due to the refractive index change in the modified area it can be observed using through light microscopy. After etching the samples are investigated again by through light microscopy and Scanning Electron Microscopy (SEM) of the facet or cross-sections to investigate the micro-channels, micro-holes and hollow volumes.

\section{Results and discussion}

The power is adjusted in a way that suitable intensities (about $\mathrm{I}=10^{13} \mathrm{~W} / \mathrm{cm}^{2}$ ) for multiphoton absorption are only available in the focal area of a tightly focused fs-laser beam. The advantage of the local modification is the freedom in the fabricated of 3D structures.

\subsection{D ISLE: Cutting}

By modifying 50 tracks in the volume of sapphire on top of each other (Fig. 5 right) and adjacent etching it is possible to fabricate cuts (Fig. 5 left).

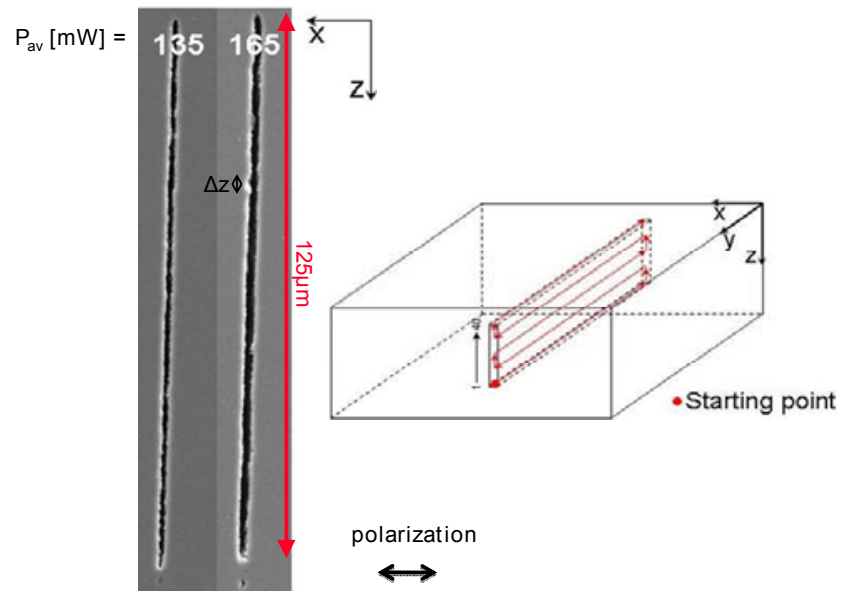

Fig. 5: Sketch of the irradiation strategy for a cut (right) and scanning electron micrographs of the cross-section of the cut existing of 50 overlapping tacks (left). Etching parameter: $\mathrm{t}=24$ hours, $c=48 \%$ HF acid.

The used repetition rate is $\mathrm{f}=500 \mathrm{kHz}$. After etching the cross-section reveals that the width of the cut kerfs is influenced slightly by the adjusted power. The dimensions (depth (y) $\mathrm{x}$ height (z) $\mathrm{x}$ width (x)) of the cuts are $1 \mathrm{~cm} \mathrm{x}$ $125 \mu \mathrm{m}$ x ca. $1 \mu \mathrm{m}$.

This process strategy can also be used to cut out 3D microparts (Fig. 6) or to fabricating microholes. The cylinder shown in (Fig. 6) left was removed from the hole (Fig. 6) right after irradiation the sapphire sample with $P=116$ $\mathrm{mW}$ and etching.

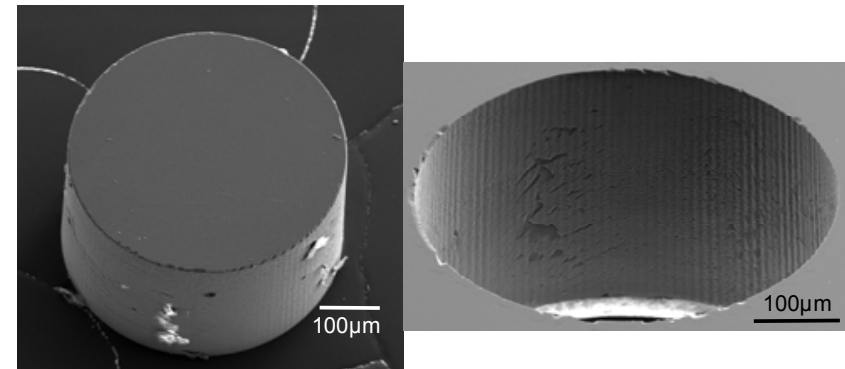

Fig. 6: SEM micrograph of a cylinder (left) with the diameter of $500 \mu \mathrm{m}$ which is fabricated with ISLE $(\mathrm{P}=116 \mathrm{~mW})$ in a $500 \mu \mathrm{m}$ thick sapphire sample. The cylinder is removed after etching ( $\mathrm{t}=$ 49 hours, $\mathrm{c}=48 \% \mathrm{HF}$ ) from the hole (right).

The cut width of the 3D microparts was investigated before removing the parts from the sample.

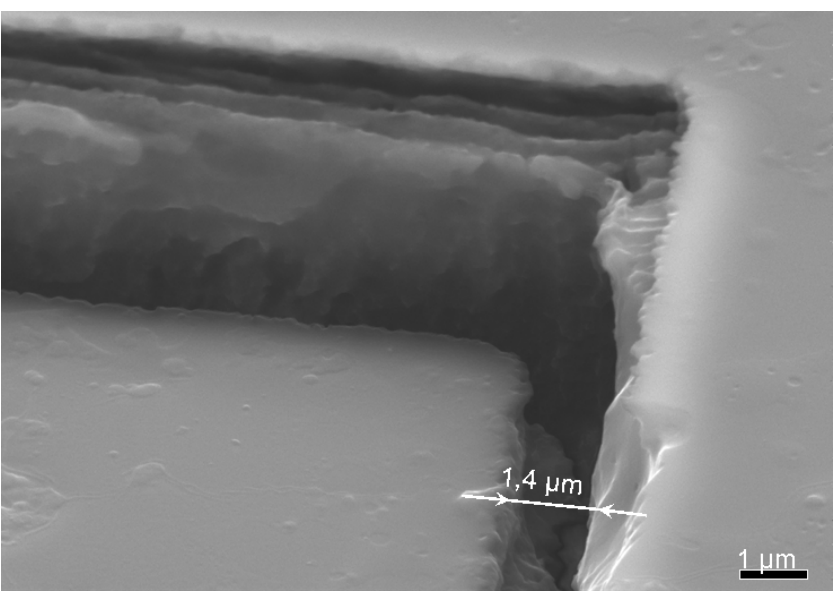

Fig. 7: SEM micrograph of the cut kerf of a cube before removing the cub from the sample. The width of the kerf is $1.4 \mu \mathrm{m}$ and the depth is $500 \mu \mathrm{m}$.

In Fig. 7 the $1.4 \mu \mathrm{m}$ wide cut kerf of a cube with the dimensions $450 \mu \mathrm{m} \times 500 \mu \mathrm{m} \times 500 \mu \mathrm{m}$ is shown. The volume of the kerf (450 $\mu \mathrm{m}$ x $2000 \mu \mathrm{m}$ x $1.4 \mu \mathrm{m})$ compared to the volume of the cube shows that the material losses is less then $2 \%$.

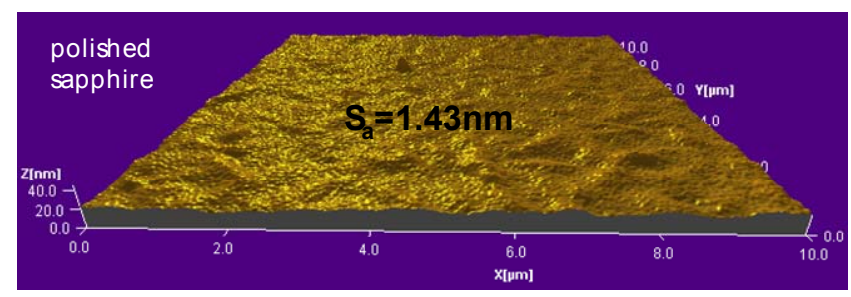

Fig. 8: AFM measurement: 3D amplitude image (10 $\mu \mathrm{m} \times 10$ $\mu \mathrm{m})$ of the optical polished surface. The average roughness $S_{a}$ is in the low nm-range $\left(\mathrm{S}_{\mathrm{a}}=1.43 \mathrm{~nm}\right)$ 


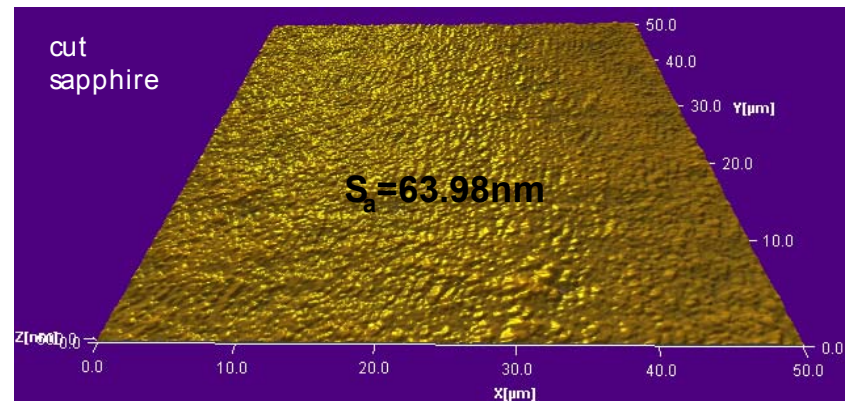

Fig. 9: AFM measurement: 3D amplitude image ( $50 \mu \mathrm{m} \times 50 \mu \mathrm{m})$ of the cut surface. The average roughness is $S_{a}=63.98 \mathrm{~nm}$.

The properties of the cut surface are investigated with atomic force microscopy (AFM) and compared to an optical polished surface. The average roughness $\left(\mathrm{S}_{\mathrm{a}}\right)$ of the cut surface is $S_{a}=63.98 \mathrm{~nm}$ (Fig. 9) and of the optical polished surface is $S_{a}=1.43 \mathrm{~nm}$ (Fig. 8). Periodical nanostructures (ripples) seem to limit the smoothness. In Fig. 7 ripples are clearly visible on the surface next to the modified and etched area. Sub-wavelength ripples are a byproduct of fslaser modification or ablation of various materials. Periodical structures are not only existing on the surface but also existing in the volume of the sample [11]. In the volume they are known as nanoplanes [12].

The orientation of ripples and nanoplanes is known to depend perpendicular on the polarization of the used laser radiation. The side of the cut cube which is parallel to the ripples (perpendicular to the polarization) might also have a different average surface roughness (lower) than the side perpendicular to the ripples. This presumption has to be clarified in following investigations.

\subsection{D ISLE: Hollow Channels}

By modifying single tracks in the volume of sapphire and adjacent etching micro channels can be produced. Micro channels are written perpendicular to the polarization of the laser. The dimension of the micro channels can be influenced by writing several tracks next to each other.

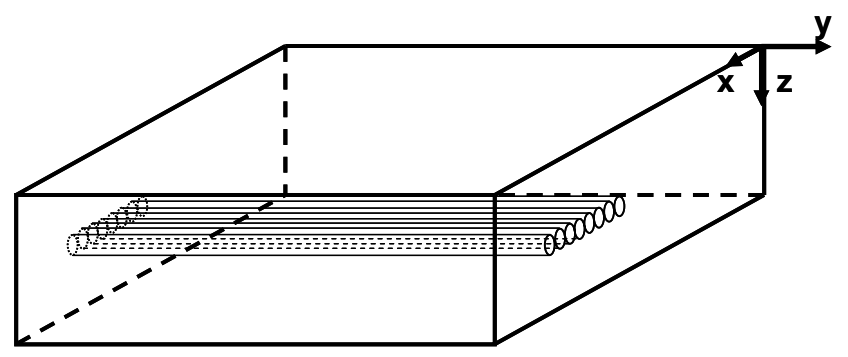

Fig. 10: Sketch of the irradiation strategy for a micro channel by modifying several adjacent tracks.

For 6 tracks with an overlap of the tracks of about $0.77 \mu \mathrm{m}$ (x-direction) and a power of $\mathrm{P}=91.3 \mathrm{~mW}$ the nearly square like shape of the cross-section of the channel shown in Fig. 11 results. The tracks are written from the right to the left side. In the first track the material is deeper modified than in the following tracks. This can be explained with reflection and scattering of the laser beam from the second track at the lower refractive index area, the modified area, of the first track.

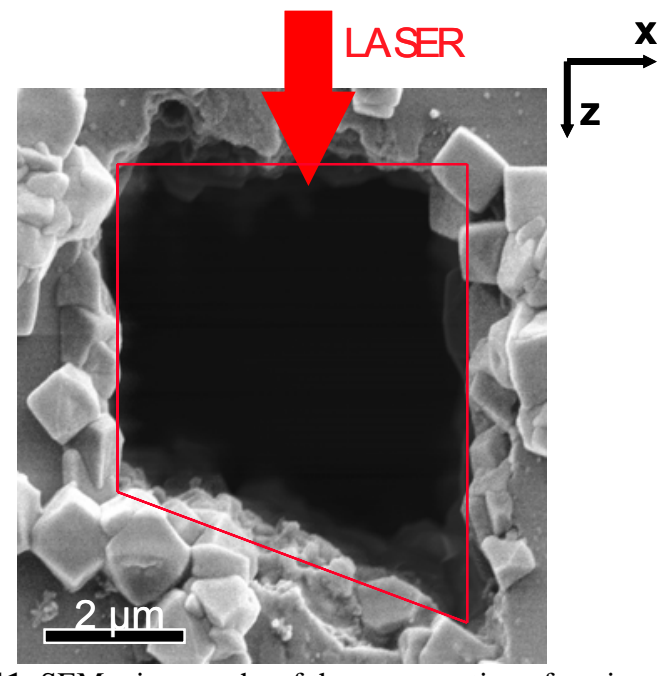

Fig. 11: SEM micrographs of the cross-section of a micro channel existing of 6 modified tracks $(\mathrm{P}=91.3 \mathrm{~mW}, \mathrm{f}=500 \mathrm{kHz}, \Delta \mathrm{x}=0.77$ $\mu \mathrm{m})$.

Depending on the adjusted power $\mathrm{P}$ and the used distance $\Delta \mathrm{x}$ of the tracks the length of the channels in y-direction varies (Fig. 12).

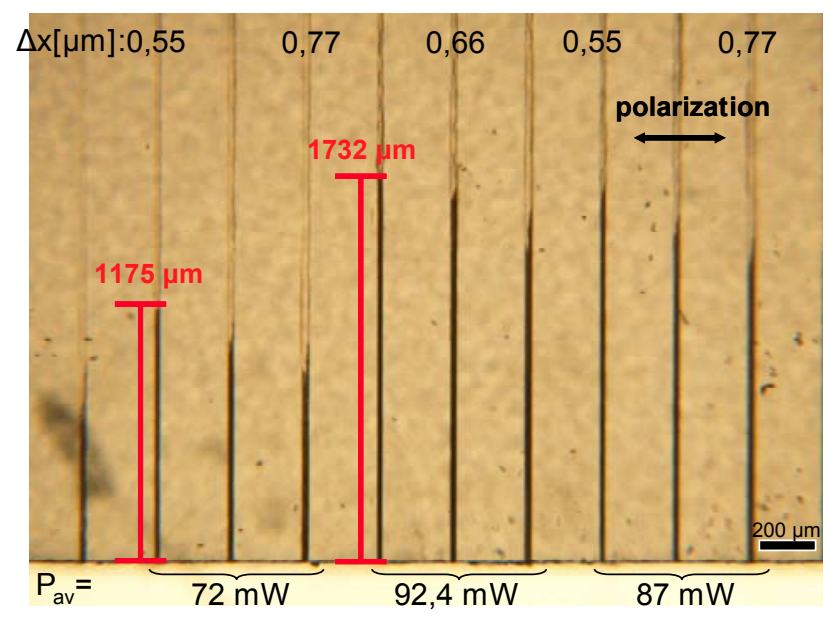

Fig. 12: Through light microscopy image showing micro channels produced with different powers and line distances $\Delta \mathrm{x}$. The micro channels existing out of 40 irradiated tracks.

The longest channel $(1732 \mu \mathrm{m})$ consisting of 40 tracks resulting with a distance of $\Delta \mathrm{x}=0.55 \mu \mathrm{m}$ and $\mathrm{P}=92.4 \mathrm{~mW}$. Higher power lead to laser induced cracks at the corners of the channel cross-section. Also higher numbers of modified tracks next to each other lead to cracks. This can be explained by structural change during the modification (from crystalline to amorphous). The more channels are next to each other the higher is the induced stress due the amortization of the material.

\subsection{Hollow Free-Form Structures}

Combining the irradiation strategies used for the fabrication of hollow channels and cutting it is possible to fabricate hollow volumes. The sample is irradiated in several $\mathrm{x}$ y layers starting with the lowest layer. Micro-channels should be irradiated to ensure a contact between acid and 
modified material. In Fig. 13 the irradiation strategy is shown for a hollow cube in sapphire. The resulting dimensions of the cube are $50 \mu \mathrm{m} \times 10 \mu \mathrm{m} \times 10 \mu \mathrm{m}$.

This free-form structure is irradiated with $\mathrm{P}=120 \mathrm{~mW}$, distance of the tracks in $\mathrm{x}$-direction of $\Delta \mathrm{x}=0.55 \mu \mathrm{m}$ and in $\mathrm{z}$-direction of $\Delta \mathrm{z}=3.5 \mu \mathrm{m}$.

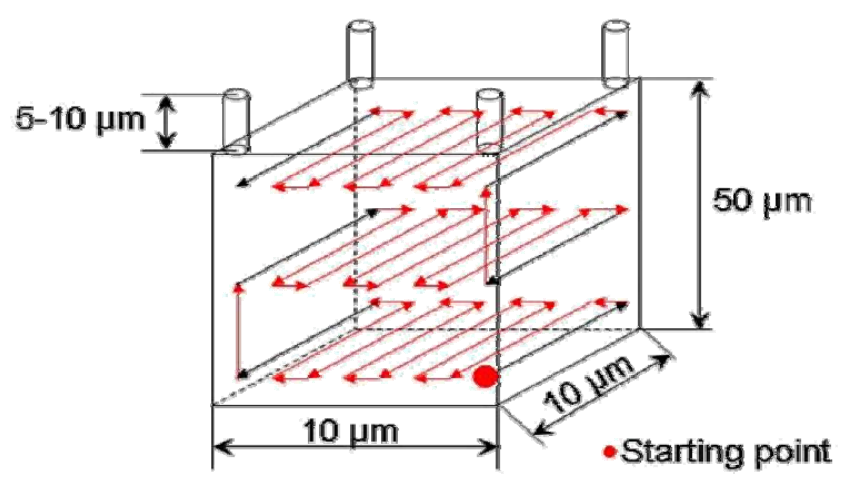

Fig. 13: Sketch of the irradiation strategy for a hollow cube.

In Fig. 14 the top view of the inlet channels is shown on the left side and the top view of the hollow volume of the cube is shown on the right side. In Fig. 14 b) residues of hollow nanoplanes can be seen on the right and the left side of the hollow volume. This shows again that the roughness of surfaces produced with ISLE is limited by periodical nanostructures.
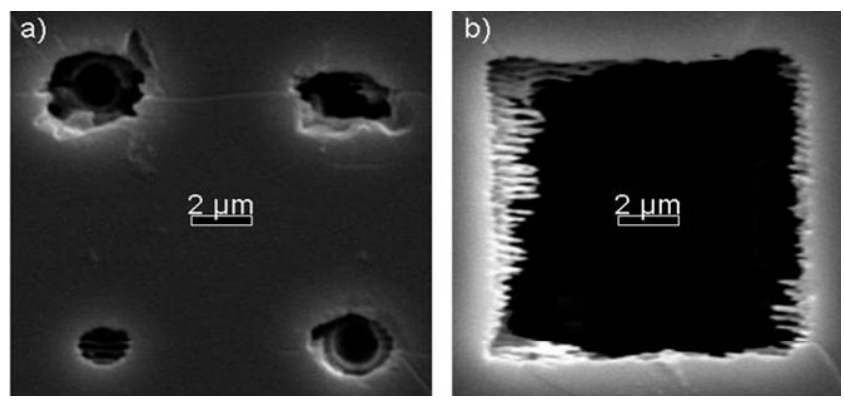

Fig. 14: SEM micrographs: Top view of the inlet channels (left), top view of the hollow volume (right).

\section{Conclusion}

ISLE is a two step process that is suitable for the 2D and $3 \mathrm{D}$ processing of sapphire. It is shown that micro parts can be removed from the sample with material losses of less than $2 \%$ of the whole volume. The average surface roughness of the cut surface is already about $\mathrm{S}_{\mathrm{a}}=64 \mathrm{~nm}$. This is a value that is already suitable for mechanical use, e.g. in bearings. It seems that periodical surface and volume structures limit the smoothness of the cut face in one direction. This problem maybe could be solved by turning the polarization or use circular polarized laser radiation.

Moreover it has been demonstrated that the production of hollow volumes is possible in sapphire.

\section{Acknowledgments and Appendixes}

We would like to thank IMRA America for the possibility to use the $\mu$ Jewel laser in the Premier Ablations Lab at the LLT.

\section{References}

[1] Y. Bellouard, A. Said, M. Dugan, and P. Bado, Fabrication of high-aspect ratio, Micro-fluidic channels and tunnels using femtosecond laser pulses and chemical etching, Optics Express., No.10, Vol. 12, pp. 21202129, (2004).

[2] S. Juodkazis, K. Nishimura, H. Misawa, T. Ebisui, R. Waki, S. Matsuo, and T. Okada, Control over the crystalline state of sapphire, Adv. Mater., 18., pp. 13611364, (2006).

[3] S. Juodkazis, Y Nishi, and H. Misawa, Femtosecond laser-assisted formation of channels in sapphire using $\mathrm{KOH}$ solution, phys.stat.sol., DOI 10.1002/ pssr.200802203, (2008)

[4] S. Juodkazis and H. Misawa, Forming tiny 3D structures for micro- and nanofluidics, SPIE Newsroom 10.1117/2.1200612.0510 (2006).

[5] Bellouard, A. Said and P. Bado, Integrated optics and mechanics in a single substrate: a step towards monolithic integration in fused silica, Opt. Express 13, 6635-6644 (2005)

[6] S. Matsuo, Y. Shichijo, T. Tomita, and S. Hashimoto, Laser fabrication of Ship-in-a-bottle Microstructures in Sapphire, JLMN 2, 114-116 (2007)

[7] K. Sugioka, Y. Cheng, And K.Midorikawa, Threedimensional micromachining of glass using femtosecond laser for lab-on-a-chip device manufacturing, Appl. Phys. A 81, 1-10 (2005).

[8] C. Hnatovsky, R. S. Taylor, E. Simova, P.P. Rajeev, D. M. Rayner, V. R. Bhardwaj, and P. B. Corkum, Fabrication of microchannels in glass using femto-second laser radiation and selective chemical etching, Appl. Phys. A 84, 47-61 (2006)

[9] S. Juodkazis, K. Nishimura, H. Misawa, and Y. Nishi, Control of the crystalline state of the sapphire, Adv. Mater. 18, 1361 (2006)

[10] M. Mazuli, S. Juodkazis, T. Ebisui, S. Matsuo, and H. Misawa, Structural characterization of shock-affected sapphire, Appl. Phys. A, 86, 197-200 (2007)

[11] J. Gottmann. D. Wortmann, and M. HörstmannJungemann, Fabrication of sub-wavelength surface ripples and in-volume nanostructures by fs-laser induced selective etching, Appl. Surface Science, 255, 5641-5646 (2008)

[12] C. Hnatovsky, R.S. Taylor, E. Simova, V.R. Bhardwaj, D.M. Rayner, P.B. Corkum, J.Appl. Phys. 98 (2005) 013517.

[13] D. Wortmann, J. Gottmann, N. Brandt, and H. HornSolle, Micro- and nanostructuring inside sapphire by fs-laser irradiation and selective etching,_ OPTICS EXPRESS Vol. 16, No. 3, 1517-1522 (2008) 